TITLE:

\title{
Metal-insulator transition, spin gap generation, and charge ordering in geometrically frustrated electron systems
}

\section{$\operatorname{AUTHOR}(\mathrm{S}):$}

Fujimoto, $S$

\section{CITATION:}

Fujimoto, S. Metal-insulator transition, spin gap generation, and charge ordering in geometrically frustrated electron systems. PHYSICAL REVIEW B 2003, 67(23): 235102.

\section{ISSUE DATE:}

2003-06-15

URL:

http://hdl.handle.net/2433/50020

RIGHT:

Copyright 2003 American Physical Society 


\title{
Metal-insulator transition, spin gap generation, and charge ordering in geometrically frustrated electron systems
}

\author{
Satoshi Fujimoto \\ Department of Physics, Kyoto University, Kyoto 606-8502, Japan
}

(Received 24 February 2003; published 10 June 2003)

\begin{abstract}
We investigate a (semi-)metal to insulator transition (MIT) realized in geometrically frustrated electron systems on the basis of the Hubbard model on a three-dimensional pyrochlore lattice and a two-dimensional checkerboard lattice. Using the renormalization-group method and mean-field analysis, we show that in the half-filling case, MIT occurs as a result of the interplay between geometrical frustration and electron correlation. In the insulating phase, which has a spin gap, the spin rotational symmetry is not broken, while charge ordering exists. The charge ordered state is stabilized so as to relax the geometrical frustration in the spin degrees of freedom. We also discuss the distortion of the lattice structure caused by the charge ordering. The results are successfully applied to the description of the MIT observed in the pyrochlore system $\mathrm{Tl}_{2} \mathrm{Ru}_{2} \mathrm{O}_{7}$.
\end{abstract}

DOI: 10.1103/PhysRevB.67.235102

PACS number(s): 71.27.+a, 71.30.+h, 75.10.Lp

\section{INTRODUCTION}

Geometrical frustration in both localized and itinerant electron systems is an important ingredient giving rise to rich variety of condensed phases. ${ }^{1-8}$ In localized spin systems, magnetic frustration suppresses a tendency toward a conventional long-range order, and may stabilize some exotic state such as a spin liquid or a valence bond crystal, as has been extensively explored both experimentally ${ }^{1-8}$ and theoretically. $^{9-25}$ Also, for itinerant systems, it has been argued that geometrical frustration may cause some novel phenomena such as heavy fermion state, ${ }^{26-32}$ anomalous Hall effect induced by the chiral order, ${ }^{33,34}$ and so forth. Among them, metal-to-insulator transition (MIT) in geometrically frustrated systems is an intriguing unsettled issue. It has been found experimentally that the pyrochlore oxides, $\mathrm{Tl}_{2} \mathrm{Ru}_{2} \mathrm{O}_{7}$ and $\mathrm{Cd}_{2} \mathrm{Os}_{2} \mathrm{O}_{7}$, and the spinel compounds, $\mathrm{CuIr}_{2} \mathrm{~S}_{4}$ and $\mathrm{MgTi}_{2} \mathrm{O}_{4}$, exhibit MIT without magnetic long-range order at finite critical temperatures. ${ }^{35-38}$ Since such systems possess the fully frustrated lattice structure, referred to as a network of corner-sharing tetrahedra (that is, a pyrochlore lattice), the magnetic properties of the insulating phase are not yet understood. Moreover, the mechanism of the MIT's observed in these systems is still an open problem. In contrast to the localized spin systems, the presence of charge degrees of freedom provides a route for the relaxation of magnetic frustration. However, when electron correlation is sufficiently strong, the magnetic frustration may still affect the lowenergy properties significantly. Thus, it is expected that geometrical frustration plays an important role in the MIT's. From this point of view, in the present paper, we study the interplay between electron correlation and geometrical frustration in the Hubbard model on a three-dimensional (3D) pyrochlore lattice and on a two-dimensional (2D) checkerboard lattice, the so-called 2D pyrochlore (Fig. 1). Although real pyrochlore oxides and spinel compounds have electronic structure composed of $t_{2 g}$ orbitals, the present study on these simpler single-band models may provide important insight into the role of geometrical frustration in MIT. Furthermore, $\mathrm{Tl}_{2} \mathrm{Ru}_{2} \mathrm{O}_{7}$ has, apart from the $t_{2 g}$ band, a nearly half-filled $\mathrm{Tl}$ $s$ band, whose important features are described by the 3D pyrochlore Hubbard model. ${ }^{39}$ We believe that this model may provide a useful understanding of the MIT undergone in this material.

The noninteracting energy bands of these two Hubbard models have a common interesting feature: They consist of a flat band (or two degenerate flat bands) on the upper band edge and a dispersive band that is tangent to the flat band (or flat bands) at the $\Gamma$ point. ${ }^{40}$ Itinerant ferromagnetism in the case that the flat bands are partially occupied has been extensively studied by several authors so far. ${ }^{40,41}$ This particular band structure is due to the geometrical property of line graph in which the above 2D and 3D pyrochlore lattices are classified. According to a theorem of the graph theory, ${ }^{42}$ a tight-binding model on a line graph generated from a bipartite graph with $V$ vertices and $E$ edges has flat bands with the degeneracy $D_{f}=\lim _{N \rightarrow \infty}(E-V+1) / N$ lying on the band edge of dispersive bands. Here, $N$ is the total number of unit cells on the lattice. It should be noted that the existence of the flat bands is analogous to the macroscopically large degeneracy of the ground state of classical spin systems on line graphs. ${ }^{9}$ Since line graphs consist of a network of complete graphs which have a frustrated structure, the presence of the flat bands is a result of geometrical frustration inherent in the lattice structure. We would like to stress that, to study effects of geometrical frustration in correlated electron systems, we should distinguish two classes of geometrically frustrated systems: one is the class of line graph and the other is not, and does not possess flat bands. For example, a triangular lattice is classified in the latter class. In this paper, we are concerned with the former class, and show that, in the halffilling case, the geometrical frustration in the above models drives the system into an insulating state with a spin gap as well as a charge gap. Also, in the insulating state, the spin rotational symmetry is not broken, while charge ordering exists. The results of this paper have been partially reported in Ref. 43 before.

The paper is organized as follows. In Sec. II, we introduce the model and present briefly some basic results derived by perturbative expansion in terms of electron-electron interac- 


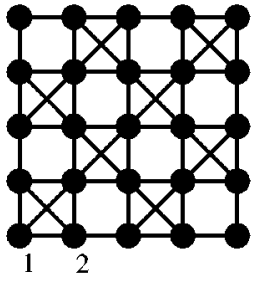

2D Checkerboard Pyrochlore

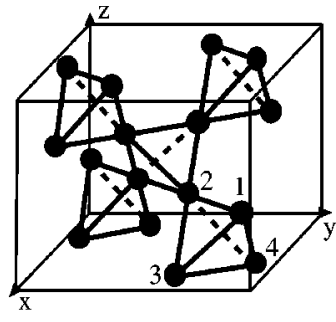

3D Pyrochlore Lattice
FIG. 1. 2D and 3D pyrochlore lattices.

tion. In Sec. III, the renormalization-group method applied to the pyrochlore Hubbard models is developed. The results for the 2D and 3D cases are given in Secs. IV and V, respectively. In particular, we discuss the (semi-)metal-to-insulator transition caused by geometrical frustration, and the properties of the insulating state in which both spin and charge excitations have a gap. We confirm our results with the use of the mean-field analysis. The effect of the coupling with lattice degrees of freedom in the presence of the charge ordering is also discussed. Summary is given in Sec. VI.

\section{MODEL HAMILTONIANS AND SOME PERTURBATIVE RESULTS}

Our system is described by the single-band Hubbard model defined on the 2D checkerboard lattice or the 3D pyrochlore lattice shown in Fig. 1. Diagonalizing the kinetic term, we write the Hamiltonian as

$$
\begin{aligned}
& H=\sum_{\mu=1}^{m} \sum_{k \sigma} E_{k \mu} a_{k \mu \sigma}^{\dagger} a_{k \mu \sigma}+\frac{U}{N} \sum_{k, k^{\prime}, q} \sum_{\alpha \beta \gamma \delta} \\
& \quad \times \Gamma_{\alpha \beta \gamma \delta}^{0}\left(k-q, k^{\prime}+q ; k^{\prime}, k\right) a_{k-q \alpha \uparrow}^{\dagger} a_{k^{\prime}+q \beta \downarrow}^{\dagger} a_{k^{\prime} \gamma \downarrow} a_{k \delta \uparrow}, \\
& \Gamma_{\alpha \beta \gamma \delta}^{0}\left(k_{1}, k_{2} ; k_{3}, k_{4}\right)=\sum_{\nu=1}^{m} s_{\nu \alpha}\left(\boldsymbol{k}_{1}\right) s_{\nu \beta}\left(\boldsymbol{k}_{2}\right) s_{\nu \gamma}\left(\boldsymbol{k}_{3}\right) s_{\nu \delta}\left(\boldsymbol{k}_{4}\right),
\end{aligned}
$$

where $m=2$ in the 2D case and $m=4$ in the 3D case. $a_{k \mu \sigma}$ $\left(a_{k \mu \sigma}^{\dagger}\right)$ is the annihilation (creation) operator for electron with momentum $k$ and spin $\sigma$ in the $\mu$ band.

In the $2 \mathrm{D}$ case

$$
\begin{gathered}
E_{k 1}=2, \\
E_{k 2}=4 \cos k_{x} \cos k_{y}-2, \\
s_{11}(\boldsymbol{k})=s_{22}(\boldsymbol{k})=\frac{\sin \left[\left(k_{x}+k_{y}\right) / 2\right]}{\sqrt{1-\cos k_{x} \cos k_{y}}}, \\
s_{21}(\boldsymbol{k})=-s_{12}(\boldsymbol{k})=\frac{\sin \left[\left(k_{x}-k_{y}\right) / 2\right]}{\sqrt{1-\cos k_{x} \cos k_{y}}} .
\end{gathered}
$$

In the $3 \mathrm{D}$ case

$$
E_{k 1}=E_{k 2}=2,
$$

$$
\begin{gathered}
E_{k 3}=-2+2 \sqrt{1+t_{k}}, \\
E_{k 4}=-2-2 \sqrt{1+t_{k}}, \\
t_{k}=\cos \left(2 k_{x}\right) \cos \left(2 k_{y}\right)+\cos \left(2 k_{y}\right) \cos \left(2 k_{z}\right) \\
+\cos \left(2 k_{z}\right) \cos \left(2 k_{x}\right) .
\end{gathered}
$$

Using the abbreviation, $s(x \pm y) \equiv \sin \left(k_{x} \pm k_{y}\right)$, etc., we write down $s_{\mu 1}, s_{\mu 2}(\boldsymbol{k})$ in the $3 \mathrm{D}$ case

$$
\begin{aligned}
& \left(s_{11}(k), s_{21}(k), s_{31}(k), s_{41}(k)\right) \\
& \quad=(s(x+z), s(y-z),-s(x+y), 0) / n_{+},
\end{aligned}
$$

where $n_{ \pm}=\sqrt{s(x \pm z)^{2}+s(y \mp z)^{2}+s(x+y)^{2}}$ and

$$
\begin{aligned}
&\left(s_{12}(k), s_{22}(k), s_{32}(k), s_{42}(k)\right) \\
&=\left(s(x+z) s(x-z) s(y-z)-s(y+z)\left[s(y-z)^{2}\right.\right. \\
&\left.+s(x+y)^{2}\right], s(x+z) s(y+z) s(y-z)-s(x-z) \\
& \times\left[s(x+z)^{2}+s(x+y)^{2}\right], \\
&-s(x+y)[s(x+z) s(y+z) \\
&\left.+s(x-z) s(y-z)], s(x+y) n_{+}^{2}\right) / n_{2},
\end{aligned}
$$

for $k_{x}+k_{y} \neq 0$, where $n_{2}=n_{+}\left(n_{+}^{2} n_{-}^{2}-[s(x+z) s(y+z)\right.$ $\left.+s(x-z) s(y-z)]^{2}\right)^{1 / 2}$ and

$$
\begin{aligned}
\left(s_{12}(\right. & \left.k), s_{22}(k), s_{32}(k), s_{42}(k)\right) \\
= & (-s(2 x),-s(2 x), 2 s(x-z), 2 s(x+z)) \\
& \times 1 / \sqrt{2 s(2 x)^{2}+4 s(x-z)^{2}+4 s(x+z)^{2}},
\end{aligned}
$$

for $k_{x}+k_{y}=0$. The expressions of $s_{\mu 3}(k)$ and $s_{\mu 4}(k)$ are very complicated. However in the following, we need only $s_{\mu 3}(k)$ for small $k$, which is given by

$$
\begin{aligned}
\left(s_{13}(k), s_{23}(k), s_{33}(k), s_{43}(k)\right) & \\
= & \left(-k_{x}-k_{y}+k_{z}, k_{x}+k_{y}+k_{z},\right. \\
& \left.\quad-k_{x}+k_{y}-k_{z}, k_{x}-k_{y}-k_{z}\right) / 2|k| .
\end{aligned}
$$

The annihilation operator of electrons at the $\mu$ th site in a unit cell is given by $c_{k \mu \sigma}=\sum_{\nu=1}^{m} s_{\mu \nu}(\boldsymbol{k}) a_{k \nu \sigma}$.

As mentioned in the Introduction, these systems have the flat band(s), $E_{k 1}$ for the 2D case, and $E_{k 1}, E_{k 2}$ for the 3D case. In the half-filling case, $n=1$, on which we concentrate henceforth, the flat band(s) is empty, while the dispersive band(s) below the flat band(s) is fully occupied. In the noninteracting half-filling case, the system is in a semimetal state, since the Fermi velocity is vanishing, though there is no excitation gap. In the following, we study how this state is affected by electron correlation.

As shown in Ref. 27, at the half filling, the perturbative calculation in $U$ for Hamiltonian (1) suffers from divergences of the single-particle self-energy, due to the presence of the flat band(s). In the 2D case, the perturbative expansion of the self-energy gives

$$
\operatorname{Re} \Sigma^{2 \mathrm{D}}(\varepsilon) \sim \text { const }+c U^{2} \ln (8 t / \varepsilon)+\cdots .
$$


In the $3 \mathrm{D}$ case, we have

$$
\begin{aligned}
\operatorname{Re} \Sigma^{3 \mathrm{D}}(\varepsilon) \sim \operatorname{const} & +c_{2} U^{2} \sqrt{|\varepsilon|}+c_{3} U^{4} / \sqrt{|\varepsilon|}+c_{4} U^{4} /|\varepsilon|^{3 / 2} \\
& +\cdots .
\end{aligned}
$$

These singular behaviors imply that some instability may be induced by electron correlation. To pursue this possibility, we will carry out the resummation of divergent terms using the renormalization-group method in the following sections.

\section{RENORMALIZATION-GROUP EQUATIONS}

To treat the infrared divergences appeared in perturbative expansion in a controlled manner, we exploit the renormalization-group (RG) method. In previous application of the RG method to electron systems, ${ }^{44-48}$ a momentum cutoff that separates the neighborhood of the Fermi surface from the higher momentum part is introduced. However, in the presence of the flat band(s), this procedure is not applicable. To circumvent this problem, we introduce the infrared energy cutoff $\Lambda$ in the following manner:

$$
\begin{aligned}
\psi_{\mu \sigma}\left(k, \varepsilon_{n}\right)= & \psi_{\mu \sigma}^{>}\left(k, \varepsilon_{n}\right) \Theta\left(\left|\varepsilon_{n}\right|-\Lambda\right) \\
& +\psi_{\mu \sigma}^{<}\left(k, \varepsilon_{n}\right) \Theta\left(\Lambda-\left|\varepsilon_{n}\right|\right) .
\end{aligned}
$$

Here, $\psi_{\mu \sigma}(k, \varepsilon)$ is the Grassmann field corresponding to $a_{k \mu \sigma}$.

Using a standard method, ${ }^{49-52}$ we obtain the RG equations of the single-particle self-energy for electrons in the $\mu$ and $\nu$ bands, $\Sigma_{\mu \nu}(k)$, and the four-point vertex functions for electrons in the $\alpha, \beta, \gamma$, and $\delta$ bands, $\Gamma_{\alpha \beta \gamma \delta}\left(k_{1}, k_{2} ; k_{3}, k_{4}\right)$ :

$$
\frac{\partial \Sigma_{\mu \nu}^{\Lambda}(k)}{\partial \Lambda}=-\sum_{k^{\prime}} \delta\left(\left|\varepsilon_{n}^{\prime}\right|-\Lambda\right) G_{\alpha \beta}^{\Lambda}\left(k^{\prime}\right) \Gamma_{\mu \beta \nu \alpha}^{(4) \Lambda}\left(k, k^{\prime} ; k, k^{\prime}\right),
$$

$$
\begin{aligned}
\frac{\partial \Gamma_{\alpha \beta \gamma \delta}^{(4) \Lambda}\left(k_{1}, k_{2} ; k_{3}, k_{4}\right)}{\partial \Lambda}= & \sum_{k, k^{\prime}} \sum_{\mu \nu \lambda \kappa}\left[\Theta\left(\left|\varepsilon_{n}\right|-\Lambda\right) \delta\left(\left|\varepsilon_{n}^{\prime}\right|-\Lambda\right)+\Theta\left(\left|\varepsilon_{n}^{\prime}\right|-\Lambda\right) \delta\left(\left|\varepsilon_{n}\right|-\Lambda\right)\right] G_{\mu \lambda}^{\Lambda}(k) G_{\nu \kappa}^{\Lambda}\left(k^{\prime}\right) \\
& \times\left[\frac{1}{2} \Gamma_{\alpha \beta \mu \nu}^{(4) \Lambda}\left(k_{1}, k_{2} ; k, k^{\prime}\right) \Gamma_{\lambda \kappa \gamma \delta}^{(4) \Lambda}\left(k, k^{\prime} ; k_{3}, k_{4}\right) \delta_{k_{1}+k_{2}, k+k^{\prime}}\right. \\
& -\Gamma_{\alpha \mu \gamma \nu}^{(4) \Lambda}\left(k_{1}, k ; k_{3}, k^{\prime}\right) \Gamma_{\kappa \beta \lambda \delta}^{(4) \Lambda}\left(k^{\prime}, k_{2} ; k, k_{4}\right) \delta_{k_{1}+k, k_{3}+k^{\prime}} \\
& \left.+\Gamma_{\alpha \mu \delta \nu}^{(4) \Lambda}\left(k_{1}, k ; k_{4}, k^{\prime}\right) \Gamma_{\kappa \beta \lambda \gamma}^{(4) \Lambda}\left(k^{\prime}, k_{2} ; k, k_{3}\right) \delta_{k_{1}+k, k_{4}+k^{\prime}}\right] \\
& +\sum_{k} \delta\left(\left|\varepsilon_{n}\right|-\Lambda\right) G_{\mu \nu}^{\Lambda}(k) \Gamma_{\alpha \beta \mu \nu \gamma \delta}^{(6) \Lambda}\left(k_{1}, k_{2}, k, k, k_{3}, k_{4}\right) .
\end{aligned}
$$

Here, $\quad G_{\mu \nu}^{\Lambda}(k)=\left[\left(i \varepsilon_{n}-E_{k \mu}\right) \delta_{\mu \nu}-\Sigma_{\mu \nu}^{\Lambda}(k)\right]^{-1} \quad$ and $\quad k$ $=\left(i \varepsilon_{n}, k\right)$ and so forth. $\Gamma^{(6) \Lambda}$ is the six-point vertex. The first, second, and third terms on the right-hand side of Eq. (18) correspond to BCS and two zero sound (ZS and $\mathrm{ZS}^{\prime}$ ) processes, respectively, of which the diagrammatic expressions are shown in Fig. 2(a).

In the following, we investigate the RG flow of the fourpoint vertex functions up to one-loop order, and drop the six-point vertex $\Gamma^{(6) \Lambda}$ and the self-energy $\Sigma^{\Lambda}(k)$ on the right-hand side of (18). In our systems, there are six species of four-point vertices, as shown in Fig. 2(b), apart from the spin degrees of freedom and the twofold degeneracy of the flat bands in the 3D case. We assume that the momentum dependences of the four-point vertex functions are given mainly by $\Gamma^{0}\left(k_{1}, k_{2} ; k_{3}, k_{4}\right)$ in the renormalization processes. This is made explicitly by replacing $\Gamma_{a b a b}\left(k_{1}, k_{2} ; k_{3}, k_{4}\right) \quad$ with $\quad g_{1} \Gamma_{a b a b}^{0}\left(k_{1}, k_{2} ; k_{3}, k_{4}\right)$, $\Gamma_{b b b a}\left(k_{1}, k_{2} ; k_{3}, k_{4}\right)$ with $g_{4} \Gamma_{b b b a}^{0}\left(k_{1}, k_{2} ; k_{3}, k_{4}\right)$, etc. This approximation is fairly good, because in the vicinity of the $\Gamma$ point, where the most important scattering processes occur, the band structure is almost isotropic. The spin degrees of (a)

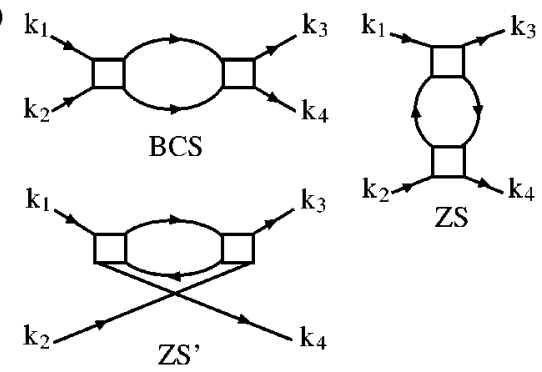

(b)
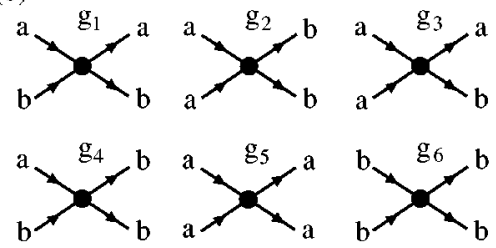

(c)

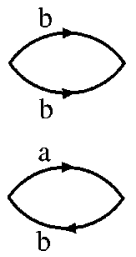

FIG. 2. BCS, ZS, and $\mathrm{ZS}^{\prime}$ processes. (b) The six species of four-point vertices. Here, " $a$ " and " $b$ " indicate the dispersive band and the flat band, respectively. (c) The leading singular bubble diagrams. 


\section{SATOSHI FUJIMOTO}

freedom of the running couplings $g_{i}(i=1 \sim 6)$ is incorporated by decomposing $g_{i}$ into the spin singlet part and the spin triplet part,

$$
\begin{aligned}
g_{i}\left(\sigma_{1}, \sigma_{2} ; \sigma_{3}, \sigma_{4}\right)= & \frac{g_{i s}}{2}\left(\delta_{\sigma_{1} \sigma_{3}} \delta_{\sigma_{2} \sigma_{4}}-\delta_{\sigma_{1} \sigma_{4}} \delta_{\sigma_{2} \sigma_{3}}\right) \\
& +\frac{g_{i t}}{2}\left(\delta_{\sigma_{1} \sigma_{3}} \delta_{\sigma_{2} \sigma_{4}}+\delta_{\sigma_{1} \sigma_{4}} \delta_{\sigma_{2} \sigma_{3}}\right) .
\end{aligned}
$$

It is also convenient in the following to decompose $g_{i}$ into the charge part and the spin part,

$$
\begin{aligned}
g_{i}\left(\sigma_{1}, \sigma_{2} ; \sigma_{3}, \sigma_{4}\right)= & \frac{3 g_{i t}-g_{i s}}{4} \delta_{\sigma_{1} \sigma_{4}} \delta_{\sigma_{2} \sigma_{3}} \\
& +\frac{g_{i s}+g_{i t}}{4} \sigma_{\sigma_{1} \sigma_{4}} \cdot \boldsymbol{\sigma}_{\sigma_{2} \sigma_{3}} .
\end{aligned}
$$

Because, at the half filling, the flat bands are empty and the dispersive band is fully occupied, the particle-particle processes between the flat bands and the particle-hole processes between the flat bands and the dispersive band give the leading singular contributions [see Fig. 2(c)]. We take into account these contributions in the derivation of the $R G$ equations. It is straightforward to show that in the vicinity of the $\Gamma$ point the following relations hold:

$$
\begin{aligned}
& \sum_{\mathrm{BCS}} \Gamma_{a a b b}^{0} \Gamma_{b b a b}^{0} \approx 0, \quad \sum_{\mathrm{ZS}} \Gamma_{a a b b}^{0} \Gamma_{b a a a}^{0} \approx 0, \\
& \sum_{\mathrm{ZS}^{\prime}} \Gamma_{a a b b}^{0} \Gamma_{b a a a}^{0} \approx 0, \quad \sum_{\mathrm{ZS}} \Gamma_{a b a b}^{0} \Gamma_{a a a b}^{0} \approx 0, \\
& \sum_{\mathrm{ZS}} \Gamma_{a b a b}^{0} \Gamma_{a a a b}^{0} \approx 0, \quad \sum_{\mathrm{ZS}} \Gamma_{a a a b}^{0} \Gamma_{b b a b}^{0} \approx 0, \\
& \sum_{\mathrm{ZS}} \Gamma_{a a b b}^{0} \Gamma_{b b b a}^{0} \approx 0, \quad \sum_{\mathrm{ZS}} \Gamma_{a a b b}^{0} \Gamma_{b b b a}^{0} \approx 0, \\
& \sum_{\mathrm{ZS}} \Gamma_{a b b b}^{0} \Gamma_{b b a b}^{0} \approx 0, \quad \sum_{\mathrm{ZS}^{\prime}} \Gamma_{a b b b}^{0} \Gamma_{b b a b}^{0} \approx 0 .
\end{aligned}
$$

Here, $\Sigma_{\mathrm{BCS}}, \Sigma_{\mathrm{ZS}}$, and $\Sigma_{\mathrm{ZS}^{\prime}}$ mean the momentum summation in the intermediate state carried out over BCS, ZS, and $\mathrm{ZS}^{\prime}$ processes, respectively. Using these relations, we find that the $\beta$ function of $g_{3}$ is approximately vanishing,

$$
\frac{d g_{3 s}}{d l} \approx \frac{d g_{3 t}}{d l} \approx 0 \text {. }
$$

and the RG equations for the other running couplings are written as ${ }^{53}$

$$
\frac{d g_{1 s}}{d l}=-\frac{a g_{4 s}^{2} e^{l}}{\Lambda_{0}}+\frac{b\left(\Lambda_{0} e^{-l}\right)^{\eta}}{4}\left(g_{1 s}^{2}+6 g_{1 s} g_{1 t}-3 g_{1 t}^{2}\right),
$$

PHYSICAL REVIEW B 67, 235102 (2003)

$$
\begin{aligned}
& \frac{d g_{1 t}}{d l}=\frac{b\left(\Lambda_{0} e^{-l}\right)^{\eta}}{4}\left(g_{1 s}^{2}-2 g_{1 s} g_{1 t}+5 g_{1 t}^{2}\right) \\
& \frac{d g_{2 s}}{d l}=-\frac{a g_{2 s} g_{6 s} e^{l}}{\Lambda_{0}}+b\left(\Lambda_{0} e^{-l}\right)^{\eta} \\
& \times\left[g_{1 s} g_{2 s}+3\left(g_{1 s} g_{2 t}+g_{1 t} g_{2 s}-g_{1 t} g_{2 t}\right)\right], \\
& \frac{d g_{2 t}}{d l}=-\frac{a g_{2 t} g_{6 t} e^{l}}{\Lambda_{0}} \\
& \frac{d g_{4 s}}{d l}=-\frac{a g_{4 s} g_{6 s}}{\Lambda_{0}} e^{l}+\frac{b\left(\Lambda_{0} e^{-l}\right)^{\eta}}{2}\left(g_{1 s} g_{4 s}+3 g_{4 s} g_{1 t}\right), \\
& \frac{d g_{4 t}}{d l}=-\frac{a g_{4 t} g_{6 t}}{\Lambda_{0}} e^{l}, \\
& \frac{d g_{5 s}}{d l}=-\frac{a g_{2 s}^{2} e^{l}}{\Lambda_{0}} \\
& \frac{d g_{5 t}}{d l}=-\frac{a g_{2 t}^{2} e^{l}}{\Lambda_{0}} \\
& \frac{d g_{6 s}}{d l}=-\frac{a g_{6 s}^{2}}{\Lambda_{0}} e^{l} \\
& \frac{d g_{6 t}}{d l}=-\frac{a g_{6 t}^{2}}{\Lambda_{0}} e^{l}
\end{aligned}
$$

where $\eta=(d-2) / 2, l=\ln \left(\Lambda_{0} / \Lambda\right)$ with $\Lambda_{0}$ the band width, and $d$ is the spatial dimension. In the $2 \mathrm{D}$ case

$$
\begin{gathered}
a=\sum_{k}\left[s_{11}^{4}(k)-s_{11}^{2}(k) s_{12}^{2}(k)\right] / 2, \\
b=\sum_{k} \operatorname{Im}\left[i \Lambda-E_{k 2}\right]^{-1} / 2 \approx 0.0622 / t,
\end{gathered}
$$

and in the $3 \mathrm{D}$ case

$$
\begin{gathered}
a=\sum_{k}\left\{\left[s_{11}^{2}(k)+s_{12}^{2}(k)\right]^{2}\right. \\
\left.-\left[s_{11}(k) s_{21}(k)+s_{12}(k) s_{22}(k)\right]^{2}\right\} / 2, \\
b=\sum_{k} \operatorname{Im}\left[i \Lambda-E_{k 3}\right]^{-1} /(4 \sqrt{\Lambda}) \approx 0.0775 / t^{3 / 2} .
\end{gathered}
$$

In the derivation of these equations for the 3D case, we have used the fact that in the vicinity of the $\Gamma$ point, the two degenerate flat bands do not mix with each other in the scattering processes. Thus, in this case, the twofold degeneracy just gives an overall factor of 2 .

Since the initial values of the running couplings in the triplet channel are zero for our models (1) the RG equations, (26), (28), (30), and (32), give $g_{2 t}=g_{4 t}=g_{5 t}=g_{6 t}=0$. In the 


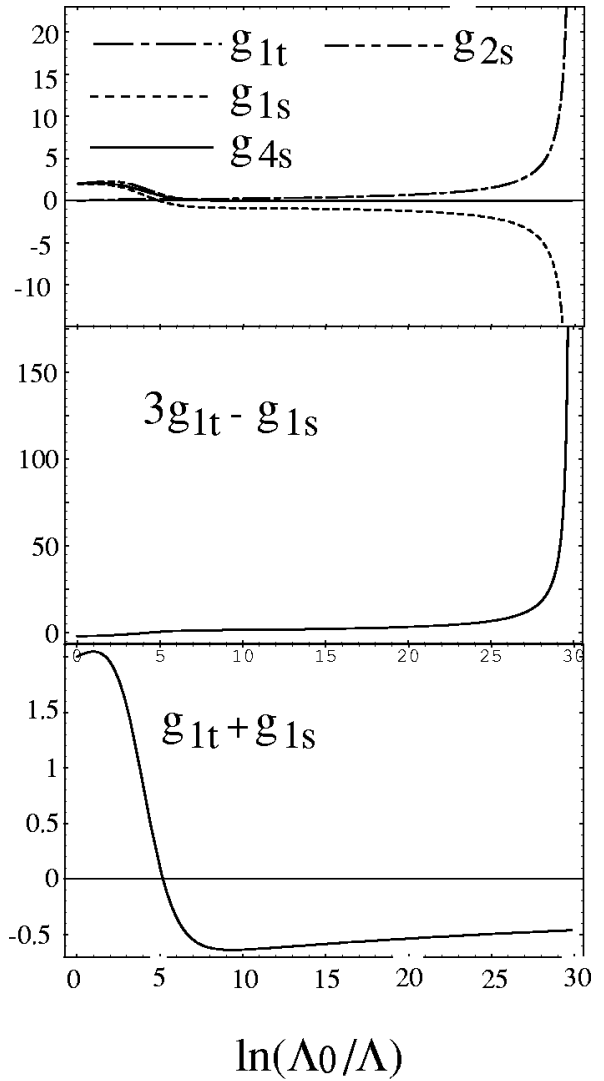

FIG. 3. The RG flow of the running couplings in the $2 \mathrm{D}$ case with $U / 8 t=0.25$.

following sections, we study how the RG flows of the other running couplings give rise to nontrivial effects.

\section{2D PYROCHLORE HUBBARD MODEL}

\section{A. RG analysis}

We first consider the 2D case, whose theoretical treatment is simpler. We solved the RG equations (23)-(32) numerically for a particular set of parameter values, and obtained the RG flow shown in Fig. 3. The running couplings $g_{5 s}$ and $g_{6 s}$, of which the RG flows are not shown in Fig. 3, are irrelevant in the low-energy limit. We found that for any small value of $U / t, g_{1 t}$ flows into the strong-coupling regime. This indicates some instability in this channel. Although $g_{1 s}$ also scales into the strong-coupling regime, it is subdominant compared to $g_{1 t}$. We also show in Fig. 3 the RG flows of the couplings $3 g_{1 t}-g_{1 s}$ and $g_{1 s}+g_{1 t}$, which are related to the charge and spin susceptibilities, respectively. We see that some instability appears in the charge degrees of freedom. To elucidate the nature of this instability more precisely, we explore how this singularity affects the single-particle self-energy. Although the diagonal parts of the self-energy $\Sigma_{11}, \Sigma_{22}$ give just a chemical potential shift up to the one-loop level, the off-diagonal self-energy $\Sigma_{12}$ changes the electronic state drastically, as will be shown below. Neglecting the diagonal self-energy, which are not im- portant in the following argument, we expand the RG equation for the off-diagonal self-energy (17) up to the first order in $\Sigma_{12}^{\Lambda}$ :

$$
\begin{gathered}
\frac{d\left(\Sigma_{12 \uparrow \uparrow}^{\Lambda}+\Sigma_{12 \downarrow \downarrow}^{\Lambda}\right)}{d l}=2 b\left(3 g_{1 t}-g_{1 s}\right)\left(\Sigma_{12 \uparrow \uparrow}^{\Lambda}+\Sigma_{12 \downarrow \downarrow}^{\Lambda}\right), \\
\frac{d\left(\Sigma_{12 \uparrow \uparrow}^{\Lambda}-\Sigma_{12 \downarrow \downarrow}^{\Lambda}\right)}{d l}=2 b\left(g_{1 s}+g_{1 t}\right)\left(\Sigma_{12 \uparrow \uparrow}^{\Lambda}-\Sigma_{12 \downarrow \downarrow}^{\Lambda}\right), \\
\frac{d \Sigma_{12 \uparrow \downarrow}^{\Lambda}}{d l}=2 b\left(g_{1 s}+g_{1 t}\right) \Sigma_{12 \uparrow \downarrow}^{\Lambda} .
\end{gathered}
$$

Because the strongest divergence of the four-point vertex appears in $3 g_{1 t}-g_{1 s}$ (see Fig. 3), the off-diagonal selfenergy $\Sigma_{\sigma} \Sigma_{12 \sigma \sigma}$ becomes nonzero at some critical $\Lambda=\Lambda_{c}$. This is easily seen by solving Eq. (37), which gives

$$
\sum_{\sigma} \Sigma_{12 \sigma \sigma}^{\Lambda}=\sum_{\sigma} \Sigma_{12 \sigma \sigma}^{\Lambda_{0}} \exp \left[2 b \int_{0}^{l} d l^{\prime}\left(3 g_{1 t}-g_{1 s}\right)\right] .
$$

Although $\Sigma_{12 \sigma \sigma}^{\Lambda_{0}}$ is vanishing in the vicinity of the $\Gamma$ point, because of the momentum dependence of $s_{\mu \nu}(k), \Sigma_{\sigma} \Sigma_{12 \sigma \sigma}^{\Lambda_{c}}$ becomes nonzero for $\Lambda=\Lambda_{c}$ at those value $3 g_{1 t}-g_{1 s}$ is divergent. The nonzero off-diagonal self-energy hybridizes the band 1 and the band 2 at the $\Gamma$ point, and drives the system into the insulating state with both spin and charge gaps. Thus, the singularity of the RG flow signifies the (semi)metal-to-insulator transition.

\section{B. Mean-field analysis}

The above RG analysis implies the existence of a meanfield solution for which the order parameter is given by

$$
\Delta_{k} \equiv \sum_{\sigma} \Sigma_{12 \sigma \sigma}(k) \sim \sum_{\sigma=\uparrow \downarrow}\left\langle a_{k 1 \sigma}^{\dagger} a_{k 2 \sigma}\right\rangle .
$$

This state is characterized by electron-hole pairing with parallel spins, which leads to the formation of both spin and charge gaps preserving the spin rotational symmetry. $\Delta_{k}$ is determined by the self-consistent mean-field equation, which is obtained as follows. According to the numerical analysis of the RG equations (23)-(31), $g_{4 s}$ is mainly renormalized by the first term on the right-hand side of Eq. (27). Then, the renormalized coupling $g_{4 s}$ is approximately given by RPAlike expressions. As a result, the self-consistent gap equation for $\Delta_{k}$ is expressed diagrammatically as shown in Fig. 4. The first term on the right-hand side of the gap equation gives less singular contributions than the second term. Thus, the linearized gap equation, which determines the transition temperature, is

$$
\begin{aligned}
\Delta_{k}= & \sum_{q, k^{\prime}} \Pi(k, q-k) G_{11}(q-k) G_{22}(q-k) \Pi\left(k^{\prime}, q-k\right) \\
& \times G_{11}\left(k^{\prime}\right) G_{22}\left(k^{\prime}\right) \Delta_{k^{\prime}},
\end{aligned}
$$

where $G_{\mu \mu}(k)=1 /\left(\varepsilon_{n}-E_{k \mu}\right), k=(i \varepsilon, k)$ and 


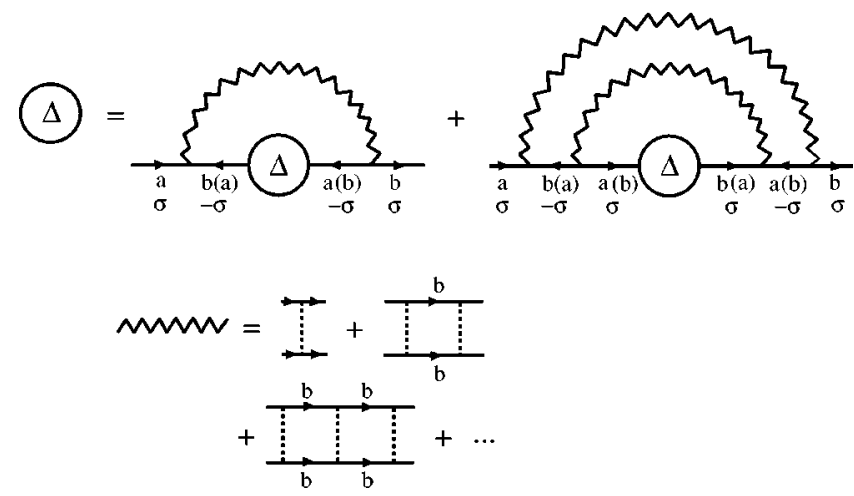

FIG. 4. Diagrams for the linearized gap equations.

$$
\begin{gathered}
\Pi\left(k, k^{\prime}\right)=\sum_{\nu= \pm} \frac{\nu U t^{\nu}\left(\boldsymbol{k}, \boldsymbol{k}^{\prime}\right)}{1-c_{\nu} D\left(k+k^{\prime}\right)}, \\
t^{ \pm}\left(\boldsymbol{k}, \boldsymbol{k}^{\prime}\right)=\left[s_{11}(\boldsymbol{k}) s_{12}\left(\boldsymbol{k}^{\prime}\right) \pm s_{12}(\boldsymbol{k}) s_{11}\left(\boldsymbol{k}^{\prime}\right)\right]^{2} / 2, \\
D(q)=-T U \sum_{n, k} G_{11}(k) G_{11}(q-k),
\end{gathered}
$$

with $c_{+}=2 a$ and $c_{-}=\Sigma_{k} s_{11}^{2}$. Here, we have ignored the diagonal self-energy. Equation (42) implies that the gap function can be written as

$$
\Delta_{k}=s_{11}(k) s_{12}(k) \Delta_{0}=\frac{\Delta_{0}\left(\cos k_{x}-\cos k_{y}\right)}{2\left(1-\cos k_{x} \cos k_{y}\right)},
$$

where $\Delta_{0}$ is a constant. From Eq. (42), we have the equation that determines the transition temperature

$$
1=\frac{U^{2}}{16 t}\left[\ln \left(\frac{8 t}{U}\right)-b_{0}\right] \ln \left(\frac{8 t}{T_{c}}\right)
$$

where $b_{0}=0.322$. Equation (47) implies that for $U<U_{c}$ $\sim 0.725(8 t)$, a state with nonzero $\Delta_{0}$ is realized. Note that the gap function (46) has a line node structure similar to $d_{x^{2}-y^{2}}$ symmetry. However, this line node vanishes, when we take into account the coupling with lattice, as will be discussed in Sec. IV D.

Generally, in 2D systems, thermal fluctuations may suppress the transition temperature down to zero. The above mean-field solution is also affected seriously by thermal fluctuations, because $U(1)$ Goldstone mode related with the phase of $\left\langle a_{k 1 \sigma}^{\dagger} a_{k 2 \sigma}\right\rangle$ does not survive at finite temperatures. To see this, we have applied the Ginzburg-Landau analysis to this mean-field solution and found that, in the $2 \mathrm{D}$ case, the transition temperature vanishes in accordance with the Mermin-Wagner-Coleman theorem. Nevertheless, the above analysis demonstrates that in the ground state at zero temperature the gap $\Delta_{k}$ is nonzero, and the system is in an insulating state.

\section{Properties of the insulating state}

\section{Spin-gap state with spin rotational symmetry}

We now further investigate the properties of the insulating phase using the mean-field solution. The single-particle Green's functions in this state are given by

$$
\begin{aligned}
& G_{11}^{\mathrm{MF}}\left(k, \varepsilon_{n}\right)=\frac{a^{(+)}}{i \varepsilon+\mu-E_{k}^{(+)}}+\frac{a^{(-)}}{i \varepsilon+\mu-E_{k}^{(-)}}, \\
& G_{22}^{\mathrm{MF}}\left(k, \varepsilon_{n}\right)=\frac{a^{(-)}}{i \varepsilon+\mu-E_{k}^{(+)}}+\frac{a^{(+)}}{i \varepsilon+\mu-E_{k}^{(-)}},
\end{aligned}
$$

where

$$
\begin{gathered}
E_{k}^{( \pm)}=\left[E_{k 1}+E_{k 2} \pm \sqrt{\left(E_{k 1}-E_{k 2}\right)^{2}+4 \Delta_{k}^{2}}\right] / 2, \\
a^{( \pm)}= \pm \frac{E^{( \pm)}-E_{k 1}}{E_{k}^{(+)}-E_{k}^{(-)}} .
\end{gathered}
$$

In the insulating phase, because the order parameter does not break the spin rotational symmetry, there is no long-range magnetic order. However, a spin-gap exists. The spin gap behavior is observed in the temperature dependence of the spin-lattice relaxation rate $1 / T_{1}$ probed by NMR measurements. It is obtained from the above mean-field solution,

$$
\begin{gathered}
\frac{1}{T_{1} T} \sim \int d E \frac{\left[N_{i}(E)\right]^{2}}{2 T \cosh ^{2} \frac{E}{2 T},} \\
N_{i}(E)=\left\langle\frac{\sqrt{E^{2}-\Delta_{k}^{2}}}{E^{2}+\Delta_{k}^{2}}\right\rangle_{k \sim 0} E^{3 / 2} .
\end{gathered}
$$

Here, $\langle\cdots\rangle_{k \sim 0}$ is the angular average near $k=0$. Because of the nodes of $\Delta_{k}$, we have $1 /\left(T_{1} T\right) \sim T^{3}$. However, as will be discussed in the following section, the coupling with lattice degrees of freedom changes this power-law behavior to an exponential decay, $1 /\left(T_{1} T\right) \sim \exp (-\Delta / T)$.

\section{Charge ordering}

Another important property of the insulating phase manifests in the charge degrees of freedom. The formation of the gap $\Delta_{k}$ brings about a difference between the charge densities at the sites 1 and 2 in a unit cell given by

$\rho_{1}-\rho_{2}=-4 \sum_{k} \frac{s_{11}(k) s_{12}(k) \Delta_{k}}{\sqrt{\left(E_{k 2}-E_{k 1}\right)^{2}+4 \Delta_{k}^{2}}} \Theta\left(\mu-E_{k}^{(-)}\right) \sim \Delta_{0} / t$.

Thus, charge ordering (CO) with a charge-density displacement proportional to the gap characterizes this insulating state. The CO pattern is shown in Fig. 5. This noteworthy result can be understood as follows. In our system, three electrons occupying nearest-neighbor sites cost energy loss caused by magnetic frustration. Conversely, magnetic frustration induces an effective finite-range repulsion between electrons at nearest-neighbor sites. If this finite-range repul- 


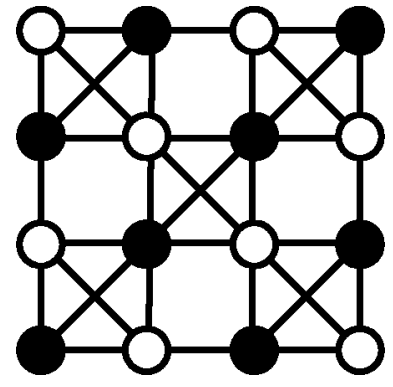

FIG. 5. The $\mathrm{CO}$ pattern in the $2 \mathrm{D}$ case.

sion is sufficiently strong to overcome the on-site Coulomb interaction $U$, the $\mathrm{CO}$ state will be stabilized. This is possible if $U$ is not so large. As $U$ increases, a transition to a conventional Mott insulator with no charge ordering should occur. This transition cannot be described within our weak-coupling analysis.

The CO pattern shown in Fig. 5 is regarded as an assembly of one-dimensional chains in the $[1,1]$ and $[1,-1]$ directions. This observation implies that $\mathrm{CO}$ reduces the spatial dimension effectively to relax geometrical frustration.

\section{Coupling with lattice distortion}

The presence of $\mathrm{CO}$ found in the preceding section implies that the coupling between the charge fluctuation and lattice degrees of freedom may give rise to the distortion of the lattice structure. Since the band structure possesses the double degeneracy at the $\Gamma$ point even for $U=0$ in the halffilling case, the Jahn-Tellar distortion may occur to lift the degeneracy. However, in our system, the lift of the double degeneracy corresponds to the situation that the charge density on the site 1 is different from that on the site 2 . It is highly nontrivial whether the inhomogeneous charge distribution is realized even in the presence of the on-site repulsion $U$, which, in general, should suppress the charge fluctuation. The results obtained in the previous sections remarkably show that the interplay between the one-site repulsion and geometrical frustration stabilizes the charge ordering in the ground state, to which the Jahn-Tellar lattice distortion may adjust. In this section, we discuss the distortion of the lattice structure compatible with the $\mathrm{CO}$ state.

Since the band degeneracy appears only at the $\Gamma$ point, it is sufficient to consider the point group of the tetragonal crystal system $D_{4 \mathrm{~h}}$. The doubly degenerate levels at the $\Gamma$ point belong to $E_{\mathrm{u}}$ representation. There are three normal modes that are relevant to the Jahn-Tellar distortion: $\left[E_{\mathrm{u}}^{2}\right]$ $=A_{1}+B_{1 \mathrm{~g}}+B_{2 \mathrm{~g}}$. The normal coordinates of these modes are, respectively, given by

$$
\begin{gathered}
Q\left(A_{1 \mathrm{~g}}\right)=-u_{1 x}-u_{1 y}+u_{2 x}-u_{2 y}+u_{3 x}+u_{3 y}-u_{4 x}+u_{4 y}, \\
Q\left(B_{1 \mathrm{~g}}\right)=u_{1 x}+u_{1 y}+u_{2 x}-u_{2 y}-u_{3 x}-u_{3 y}-u_{4 x}+u_{4 y}, \\
Q\left(B_{2 \mathrm{~g}}\right)=-u_{1 x}+u_{1 y}+u_{2 x}+u_{2 y}+u_{3 x}-u_{3 y}-u_{4 x}-u_{4 y},
\end{gathered}
$$
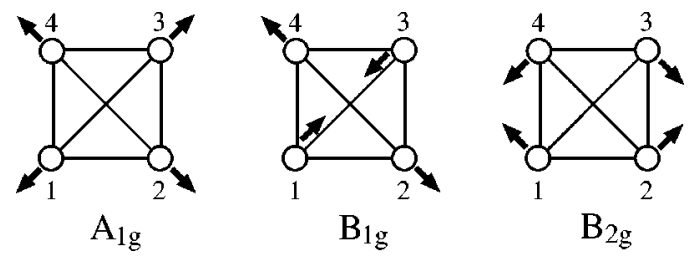

FIG. 6. Three normal modes of the lattice distortion.

where $u_{i \alpha}(i=1,2,3,4$ and $\alpha=x, y, z)$ is the displacement of the $i$ site in the $\alpha$ direction. The lattice distortions corresponding to these modes are schematically shown in Fig. 6. The lattice distortions change the kinetic term of the Hamiltonian $H_{\text {kin }} \rightarrow H_{\text {kin }}+\Delta H_{\text {kin }}$ with

$$
\begin{aligned}
\Delta H_{\mathrm{kin}}= & -a_{A_{1 \mathrm{~g}}} \sum_{i, j}\left(c_{1 i}^{\dagger} c_{1 j}+c_{2 i}^{\dagger} c_{2 j}+c_{1 i}^{\dagger} c_{2 j}+c_{2 i}^{\dagger} c_{1 j}\right) Q\left(A_{1 \mathrm{~g}}\right) \\
& -a_{B_{1 \mathrm{~g}}} \sum_{i, j}\left(c_{1 i}^{\dagger} c_{1 j}-c_{2 i}^{\dagger} c_{j}\right) Q\left(B_{1 \mathrm{~g}}\right) \\
& -a_{B_{2 \mathrm{~g}}} \sum_{i}\left(c_{1 i}^{\dagger} c_{2 i+a_{x}}-c_{1 i}^{\dagger} c_{2 i+a_{y}}+c_{2 i}^{\dagger} c_{1 i+a_{x}}\right. \\
& \left.-c_{2 i}^{\dagger} c_{1 i+a_{y}}+\text { H.c. }\right) Q\left(B_{2 \mathrm{~g}}\right) .
\end{aligned}
$$

Here, we omit the spin index. The $A_{1 \mathrm{~g}}$ and $B_{2 \mathrm{~g}}$ modes do not lift the degeneracy at the $\Gamma$ point. Obviously, the $B_{1 \mathrm{~g}}$ mode is the only lattice distortion that is consistent with the $\mathrm{CO}$ pattern shown in Fig. 5. After this lattice distortion occurs, the single-particle excitation gap completely opens at the $\Gamma$ point without line nodes, leading the exponential decay of the spin-lattice relaxation rate, as announced in the preceding section.

\section{3D PYROCHLORE HUBBARD MODEL}

\section{A. RG analysis}

The above analysis can be straightforwardly generalized to the case of a 3D pyrochlore lattice. We obtain the RG flow numerically from Eqs. (23) $-(32)$ for $d=3$. Here, in contrast to the $2 \mathrm{D}$ case, for sufficiently small $U$ all couplings are irrelevant, and thus the semimetal state is stable. However, for values of $U$ larger than a certain critical value but still smaller than the band width, RG flow similar to that in the $2 \mathrm{D}$ case is obtained, as shown in Fig. 7 . The coupling $3 g_{1 t}$ $-g_{1 s}$, which is related to the charge degrees of freedom, scales into the strong-coupling regime. This RG flow implies that, as in the 2D case, a particle-hole pairing between the flat bands and the dispersive band is realized below a critical temperature, leading spontaneous gap formation both in the charge and spin degrees of freedom. Note that the spin-gap formation does not signify the breaking of the spin rotational symmetry, since the vertex in the spin degrees of freedom $g_{1 s}+g_{1 t}$ is still finite at the critical value of $\ln \left(\Lambda_{0} / \Lambda\right)$.

\section{B. Mean-field analysis}

Although the value of $U$ used above is relatively large, we expect that the one-loop RG calculation still gives qualita- 


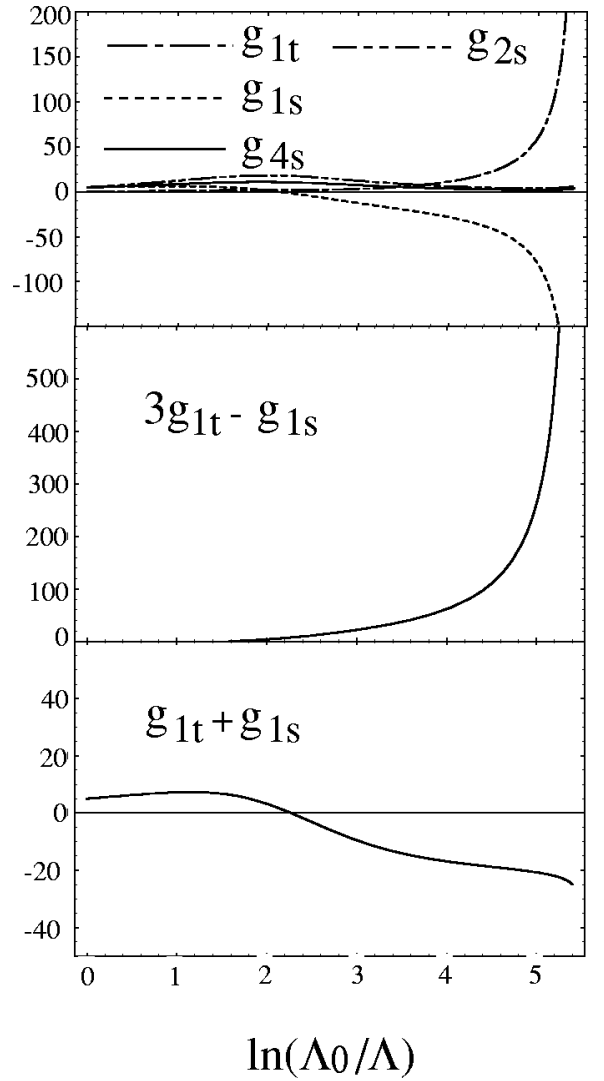

FIG. 7. The RG flow of the running couplings in the 3D case with $U / 8 t=0.625$.

tively correct results, as long as $U$ is smaller than the band width. To examine the validity of the one-loop calculation, we explore the self-consistent mean-field solution. The order parameters for the particle-hole pairing state suggested from the above RG flows is,

$$
\begin{aligned}
& \Delta_{k}^{(13)}=\sum_{\sigma}\left\langle a_{k 1 \sigma}^{\dagger} a_{k 3 \sigma}\right\rangle, \\
& \Delta_{k}^{(23)}=\sum_{\sigma}\left\langle a_{k 2 \sigma}^{\dagger} a_{k 3 \sigma}\right\rangle .
\end{aligned}
$$

The self-consistent gap equations for the 3D case are also given by the diagram shown in Fig. 4, from which we find that the gap functions are given by

$$
\begin{aligned}
\Delta_{k}^{(13)} & =\sum_{\nu=1}^{4} s_{\nu 1}(k) s_{\nu 3}(k) \Delta_{\nu}^{(13)} \\
& =\sum_{\nu=1}^{2} s_{\nu 1}(k) s_{\nu 3}(k)\left(\Delta_{\nu}^{(13)}-\Delta_{3}^{(13)}\right) \\
\Delta_{k}^{(23)} & =\sum_{\nu=1}^{4} s_{\nu 2}(k) s_{\nu 3}(k) \Delta_{\nu}^{(23)} \\
& =\sum_{\nu=1}^{3} s_{\nu 2}(k) s_{\nu 3}(k)\left(\Delta_{\nu}^{(23)}-\Delta_{4}^{(23)}\right) .
\end{aligned}
$$

Here, we used the orthogonal relations $\sum_{\nu=1}^{4} s_{\nu 1} s_{\nu 3}=0$, $\sum_{\nu=1}^{4} s_{\nu 2} s_{\nu 3}=0$, and $s_{41}=0$. Using the symmetry properties of $s_{\mu \nu}(k)$ in momentum space, we can impose some restrictions on the structure of the gap functions without solving the gap equations. Under the transformation, $x \rightarrow y, y \rightarrow x$, $z \rightarrow-z$, the coefficients of $\Delta_{k}^{(13)}$ are transformed as

$$
\begin{aligned}
& s_{11} s_{13} \rightarrow-s_{21} s_{23}, \\
& s_{21} s_{23} \rightarrow-s_{11} s_{13}, \\
& s_{31} s_{33} \rightarrow-s_{31} s_{33} .
\end{aligned}
$$

Because of the symmetry of a tetrahedron $T_{d}$, the gap function should be unchanged up to the sign by this transformation. Then, we have

$$
\Delta_{1}^{(13)}=\Delta_{2}^{(13)}
$$

or

$$
\Delta_{1}^{(13)}+\Delta_{2}^{(13)}=2 \Delta_{3}^{(13)} .
$$

In a similar manner, using the transformation $x \rightarrow x, y \rightarrow z$, $z \rightarrow y$, we obtain

$$
\Delta_{1}^{(13)}=\Delta_{3}^{(13)}
$$

or

$$
\Delta_{1}^{(13)}+\Delta_{3}^{(13)}=2 \Delta_{2}^{(13)} .
$$

Combining Eqs. (67) and (69), we end up with $\Delta_{1}^{(13)}$ $=\Delta_{2}^{(13)}=\Delta_{3}^{(13)}$ and thus,

$$
\Delta_{k}^{(13)}=0 .
$$

Applying a similar argument to $\Delta_{k}^{(23)}$, we find $\Delta_{1}^{(23)}=\Delta_{2}^{(23)}$ $=\Delta_{3}^{(23)}$ and

$$
\Delta_{k}^{(23)}=s_{42}(k) s_{43}(k)\left(\Delta_{4}^{(23)}-\Delta_{1}^{(23)}\right) .
$$

The quantity $\Delta_{4}^{(23)}-\Delta_{1}^{(23)}$ is determined from the gap equation. According to the RG analysis, the transition occurs only for sufficiently large $U$. Therefore, to determine the transition temperature and the gap function correctly, we need to take into account the self-energy corrections, i.e., pair breaking effect. This calculation is rather involved, and we have not yet carried it out. However, we see from the RG flow that at the critical temperature $T_{c} \sim \Lambda_{0} e^{-l_{c}}=(8 t) 0.0042$, a transition from a semimetal to an insulator occurs. In the resulting insulating state, the threefold degeneracy at the $\Gamma$ point in the semi-metal state is lifted completely, and a spin gap as well as a charge gap exists. The gap function $\Delta_{k}^{(23)}$ has both line and point nodes determined by $s_{42}(k) s_{43}(k)=0$. However, these nodes are eliminated by the coupling with lattice degrees of freedom, as will be shown in Sec. VD.

The particle-hole pairing state characterized by the order parameters (59) and (60) is analogous to the excitonic insulator. ${ }^{54}$ However, this analogy is not complete. In contrast to the excitonic insulator that is realized in particle-hole symmetric bands, the pairing state found above is stabilized by strong interaction between flat bands and dispersive bands 
in the absence of particle-hole symmetry. Thus, we believe that this is a mechanism for the particle-hole pairing specific to pyrochlore systems.

\section{Properties of the insulating state}

\section{Spin-gap state with spin rotational symmetry}

It is straightforward to compute magnetic properties of the gapped state using the mean-field solution obtained in the preceding section. In the insulating phase with the singleparticle excitation gap $\Delta_{k}^{(23)}$, the spin-lattice relaxation rate $1 / T_{1}$ is also given by Eq. (53). Since the gap function has nodes, $1 /\left(T_{1} T\right)$ decreases in a power law as temperature is lowered: $1 /\left(T_{1} T\right) \sim T^{3}$. However, as will be discussed in Sec. $\mathrm{VD}$, in the presence of the coupling with lattice degrees of freedom, the nodes of the gap function disappear. In this case, the spin-lattice relaxation rate shows an exponential behavior, $1 /\left(T_{1} T\right) \sim \exp (-\Delta / T)$.

The spin-gap behavior appears in the spin-spin correlation function $\operatorname{Im} \chi_{s}(q, \omega)$ for all $q$. The momentum dependence of $\operatorname{Im} \chi_{s}(q, \omega)$ is quite small, indicating that geometrical frustration suppresses any tendency toward a conventional magnetic order. Such small $q$-dependence of $\operatorname{Im}_{\chi_{s}}(q, \omega)$ was also found in the semimetal state above $T_{c} \cdot{ }^{27}$

\section{Charge ordering}

Here, we examine the possibility of a $\mathrm{CO}$ state in the 3D system. There are four sites in a unit cell. The appearance of a gap causes a charge-density displacement on each site given by

$$
\delta \rho_{\nu}=2 \sum_{k} s_{\nu 2}(k) s_{\nu 3}(k) \Delta_{k}^{(23)},
$$

for $\nu=1,2,3$, and

$$
\delta \rho_{4}=-\sum_{\nu=1}^{3} \delta \rho_{\nu}
$$

Using the symmetry properties of $s_{\mu \nu}(k)$, we obtain

$$
\begin{gathered}
\delta \rho_{1}=\delta \rho_{2}=\delta \rho_{3} \neq 0, \\
\delta \rho_{4}=-3 \delta \rho_{1} .
\end{gathered}
$$

It is thus found that $\mathrm{CO}$ with the pattern displayed in Fig. 8 occurs in the insulating phase. Interestingly, a similar $\mathrm{CO}$ pattern is observed in the spinel system $\mathrm{AlV}_{2} \mathrm{O}_{4}$ which possesses a $V$ site corner-sharing tetrahedron network. ${ }^{55}$ Within the above analysis, the sign of $\delta \rho_{4}$, which depends on the phase of the order parameter $\Delta_{4}^{(23)}-\Delta_{1}^{(23)}$, is not determined. Thus, the states of $\delta \rho_{4}>0$ and $\delta \rho_{4}<0$ are degenerated. As will be discussed in the following section, this degeneracy is lifted by the coupling with lattice degrees of freedom.

Note that the $\mathrm{CO}$ pattern shown in Fig. 8 is regarded as an alternate stack of 2D kagome lattices and triangular lattices in the $[-1,1,-1]$ direction. As in the $2 \mathrm{D}$ case, $\mathrm{CO}$ brings about the effective reduction of spatial dimension to relax geometrical frustration.

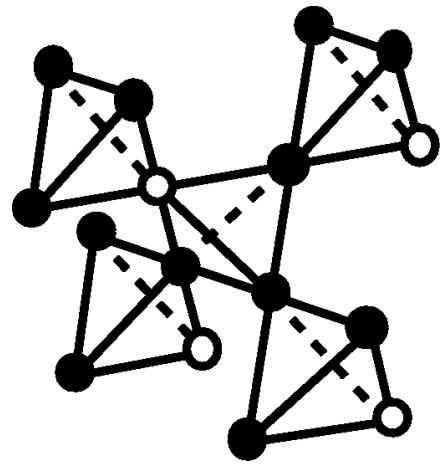

FIG. 8. Fig. 8. The $\mathrm{CO}$ pattern in the $3 \mathrm{D}$ case.

\section{Coupling with lattice distortion}

As in the 2D case, $\mathrm{CO}$ found in the preceding section may bring about lattice distortion. Here, we discuss this possibility. Since, at the half filling, all important processes occur in the vicinity of the $\Gamma$ point, it is sufficient to consider the point group of the tetrahedron $T_{\mathrm{d}}$. The representation of $T_{\mathrm{d}}$ is reduced to $A_{1}+T_{2}$. The triply degenerate levels at the $\Gamma$ point $E_{k 1}=E_{k 2}=E_{k 3}(k=0)$ belong to $T_{2}$. Thus, the normal modes that may lift the degeneracy are obtained from the symmetric product representation, $\left[T_{2}^{2}\right]=A_{1}+\mathrm{E}+T_{2}$. The normal coordinates corresponding to these three modes are

$$
\begin{gathered}
Q\left(A_{1}\right)=\left(u_{1 x}-u_{2 x}+u_{3 x}-u_{4 x}+u_{1 y}-u_{2 y}-u_{3 y}+u_{4 y}+u_{1 z}\right. \\
\left.+u_{2 z}-u_{3 z}-u_{4 z}\right) / \sqrt{3}, \\
Q^{(1)}(E)=\left(u_{1 x}-u_{2 x}+u_{3 x}-u_{4 x}+u_{1 y}-u_{2 y}-u_{3 y}+u_{4 y}\right. \\
\left.-2 u_{1 z}-2 u_{2 z}+2 u_{3 z}+2 u_{4 z}\right) / \sqrt{6},
\end{gathered}
$$

$$
\begin{aligned}
& Q^{(1)}\left(T_{2}\right)=u_{1 x}+u_{2 x}-u_{3 x}-u_{4 x}, \\
& Q^{(2)}\left(T_{2}\right)=u_{1 y}-u_{2 y}+u_{3 y}-u_{4 y}, \\
& Q^{(3)}\left(T_{2}\right)=u_{1 z}-u_{2 z}-u_{3 z}+u_{4 z}
\end{aligned}
$$

(a)

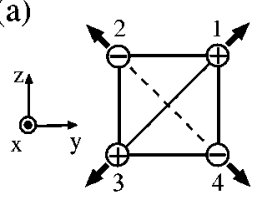

$\mathrm{A}_{1}$ (b)

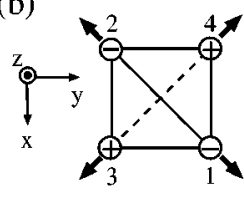

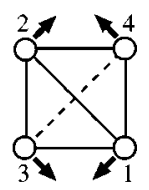

$\mathrm{E}$ (c)
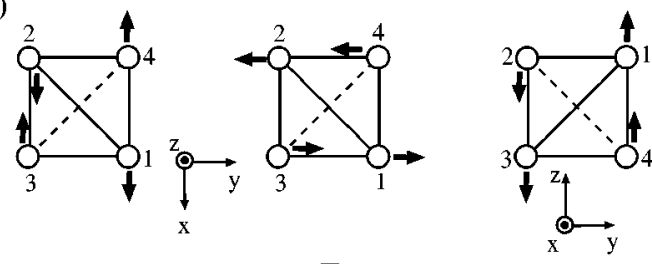

$\mathrm{T}_{2}$

FIG. 9. The normal modes of $T_{\mathrm{d}}$. (a) $A_{1}$, (b) $E$, (c) $T_{2}$. 


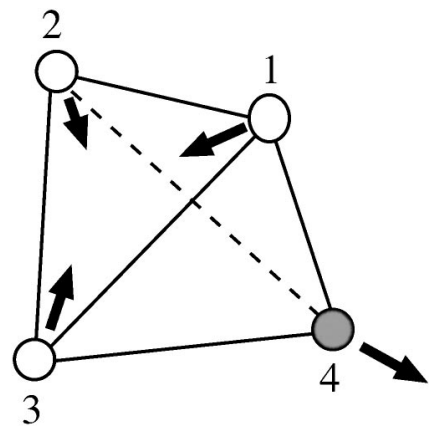

FIG. 10. The lattice distortion consistent with the $\mathrm{CO}$ pattern shown in Fig. 8.

They are schematically shown in Figs. $9(\mathrm{a}-\mathrm{c})$. The breezing mode $A_{1}$ does not lift the degeneracy at the $\Gamma$ point. The $E$ and $T_{2}$ modes split the triply degenerate levels into three different levels or one separated level and doubly degenerate levels. The modification of the kinetic energy due to these modes are

$$
\begin{aligned}
\Delta H_{\text {kin }}= & b_{E} \sum_{i, j}\left[\frac { Q ^ { ( 1 ) } ( E ) } { 2 \sqrt { 3 } } \left(2 c_{i 1}^{\dagger} c_{j 2}+2 c_{i 3}^{\dagger} c_{j 4}-c_{i 1}^{\dagger} c_{j 3}\right.\right. \\
& \left.-c_{i 2}^{\dagger} c_{j 3}-c_{i 2}^{\dagger} c_{j 4}-c_{i 1}^{\dagger} c_{j 4}+\text { H.c. }\right) \\
& \left.+\frac{Q^{(2)}(E)}{2}\left(c_{i 1}^{\dagger} c_{j 4}+c_{i 2}^{\dagger} c_{j 3}-c_{i 1}^{\dagger} c_{j 3}-c_{i 2}^{\dagger} c_{j 4}+\text { H.c. }\right)\right] \\
& +b_{T_{2}} \sum_{i, j}\left[\frac{Q^{(1)}\left(T_{2}\right)}{\sqrt{2}}\left(c_{i 1}^{\dagger} c_{j 4}-c_{i 2}^{\dagger} c_{j 3}+\text { H.c. }\right)\right. \\
& +\frac{Q^{(2)}\left(T_{2}\right)}{\sqrt{2}}\left(c_{i 1}^{\dagger} c_{j 2}-c_{i 3}^{\dagger} c_{j 4}+\text { H.c. }\right) \\
& \left.+\frac{Q^{(3)}\left(T_{2}\right)}{\sqrt{2}}\left(c_{i 1}^{\dagger} c_{j 3}-c_{i 2}^{\dagger} c_{j 4}+\text { H.c. }\right)\right] .
\end{aligned}
$$

Since the stretch of bonds may reduce the hopping integral, it is plausible to assume $b_{E}>0$ and $b_{T_{2}}>0$. The lattice distortion that is consistent with the $\mathrm{CO}$ pattern shown in Fig. 8 is obtained by putting $Q^{(1)}\left(T_{2}\right)=-Q^{(2)}\left(T_{2}\right)=-Q^{(3)}\left(T_{2}\right) \neq 0$ and $Q^{(1)}(E)=Q^{(2)}(E)=0$. This mode is shown in Fig. 10 . Here, we have dropped the rotational degrees of freedom around $(-1,1,-1)$ axis. For $Q^{(1)}\left(T_{2}\right)>0$, the triply degenerate levels at the $\Gamma$ point split into one lower level and upper doubly degenerate levels. Thus, at the half filling, this lattice distortion generates a full gap without a node in the single-particle excitation. Note that the full gap opens not only at the $\Gamma$ point but also over the entire Brillouin zone, as easily verified by diagonalizing the kinetic term with this lattice distortion. On the other hand, for $Q^{(1)}\left(T_{2}\right)<0$, the lower levels are doubly degenerated, and the system is still in the semimetallic state unless we do not take into account the correlation-driven $\mathrm{CO}$ transition obtained in the previous sections. Thus, it is expected that the case of $Q^{(1)}\left(T_{2}\right)>0$, in which both the lattice distortion and electron correlation sta- bilize the gapped state, is energetically favorable. Under the trigonal distortion with $Q^{(1)}\left(T_{2}\right)>0$ shown in Fig. 10, the charge density on the site 4 in the tetrahedron is larger than those on the other three sites. This is easily seen by calculating straightforwardly the charge density on each site in a single tetrahedron with the trigonal distortion. Then, the sign of $\delta \rho_{4}$, which is not determined in the absence of the lattice coupling as mentioned in the previous sections, is chosen as $\delta \rho_{4}>0$ by the lattice distortion.

The lattice distortion discussed here and the $\mathrm{CO}$ state shown in Fig. 8 are similar to the experimental observation for $\mathrm{AlV}_{2} \mathrm{O}_{4}$, apart from the doubling of the unit cell in the $[1,1,1]$ direction found in $\mathrm{AlV}_{2} \mathrm{O}_{4} \cdot{ }^{55}$ Since, in $\mathrm{AlV}_{2} \mathrm{O}_{4}$, the $t_{2 g}$ orbital of $V$ sites plays a central role, our simple model is not directly applicable to this system. However, according to the recent local-density approximation calculation, the band structure near the Fermi surface possesses $s$-like character because the $s$ orbital of $\mathrm{Al}$ site partially hybridizes with the $a_{1 g}$ orbital split from the $t_{2 g}$ orbital by a trigonal crystal field. ${ }^{56}$ Thus, $\mathrm{CO}$ observed in $\mathrm{AlV}_{2} \mathrm{O}_{4}$ may be microscopically explained by the mechanism described here. It should be emphasized that in our scenario, the interplay between electron correlation and geometrical frustration is the most important ingredient for the realization of the $\mathrm{CO}$ state and the lattice distortion is merely a secondary effect.

\section{E. Application to MIT in $\mathrm{Tl}_{2} \mathrm{Ru}_{2} \mathrm{O}_{7}$}

We now apply the results obtained above to the description of the MIT observed in $\mathrm{Tl}_{2} \mathrm{Ru}_{2} \mathrm{O}_{7}$. As mentioned in the Introduction, according to Ishii and Oguchi, the electronic structure of this system consists of the $s$ orbital of Tl sites, as well as the $t_{2 g}$ orbitals of $\mathrm{Ru}$ sites. ${ }^{39}$ The band structure formed by the former is well approximated by our model. The band calculation gives the band width of this system $8 t \sim 2 \mathrm{eV} .{ }^{39}$ Experimental data on the size of $U$ does not exists. However, typically, the value of $U$ for transition-metal oxides is $\sim 2 \mathrm{eV}$. This gives us reason to believe that the analysis presented in this paper, which suggests that the MIT occurs for large $U \sim 8 t$, can be applied to the description of the $\mathrm{Tl}_{2} \mathrm{Ru}_{2} \mathrm{O}_{7}$ system. The transition temperature estimated from the RG analysis is $T_{c} \sim 98 \mathrm{~K}$, which is almost comparable with the experimental values $100 \sim 120 \mathrm{~K} .{ }^{35} \mathrm{~A}$ recent NMR measurement of $\mathrm{Tl}$ nuclei has revealed the presence of a spin gap in the insulating state, which is consistent with our results. ${ }^{57}$ The possible existence of a $\mathrm{CO}$ state and large enhancement of charge fluctuations above $T_{c}$ predicted in our theory have not yet been investigated experimentally. The experimental determination of whether a $\mathrm{CO}$ state exists for $\mathrm{Tl}_{2} \mathrm{Ru}_{2} \mathrm{O}_{7}$ is a crucial test of this theory. When there exists coupling to a lattice, $\mathrm{CO}$ should accompany lattice distortion. As discussed in Sec. V C, the CO pattern found in this study suggests that the cubic lattice symmetry is broken down to trigonal symmetry. Actually, it has been found experimentally that, in $\mathrm{Tl}_{2} \mathrm{Ru}_{2} \mathrm{O}_{7}$, the lattice structure changes discontinuously at the MIT point. This observation seems to suggest the presence of large charge fluctuations in this system. 


\section{SUMMARY}

We have studied the MIT caused by the interplay between geometrical frustration and electron correlation. We have found, using the RG method and mean-field analysis that the 2D and 3D pyrochlore Hubbard models at the half-filling show the transition from semi-metal to spin-gapped insulator. The transition occurs at a finite critical temperature $T_{c}$ in the $3 \mathrm{D}$ case, and at $T=0$ in the $2 \mathrm{D}$ case. In the insulating state, $\mathrm{CO}$ occurs concomitantly so as to relax geometrical frustration. The results obtained here are successfully applied to the description of the MIT observed in the pyrochlore oxide $\mathrm{Tl}_{2} \mathrm{Ru}_{2} \mathrm{O}_{7}$, though it is a future issue to examine experimentally the presence of the $\mathrm{CO}$ state in this system, as predicted from our theory. The $\mathrm{CO}$ pattern found in this paper is also very similar to that observed in $\mathrm{AlV}_{2} \mathrm{O}_{4}$. The mechanism for $\mathrm{CO}$ in this system may be explained by the scenario described in this paper, because the electronic structure of $\mathrm{AlV}_{2} \mathrm{O}_{4}$ possesses partially flat bands, ${ }^{56}$ which is a crucial ingredient for $\mathrm{CO}$ induced by geometrical frustration. It is a future issue to explore this possibility taking into account the $t_{2 g}$ orbitals of $V$ sites.
The insulating state found in our systems is characterized by particle-hole pairing. In this sense, it is analogous to the excitonic insulator. However, there are some important differences between them. In contrast to the excitonic insulator, the spin-gapped insulator in the pyrochlore Hubbard models is stabilized by the presence of flat bands originated from geometrical frustration. Interestingly, Singh et al. pointed out a possibility of the excitonic insulator realized in the pyrochlore oxides $\mathrm{Cd}_{2} \mathrm{Os}_{2} \mathrm{O}_{7} \cdot{ }^{58}$ Since its electronic structure near the Fermi energy consists of the $t_{2 g}$ manifolds, our model is not simply applicable to this system. It is an intriguing issue to extend our analysis to more realistic models with this electronic structure.

\section{ACKNOWLEDGMENTS}

The author is grateful to K. Yamada and H. Harima for invaluable discussions. This work was supported by a Grantin-Aid from the Ministry of Education, Science, and Culture, Japan.
${ }^{1}$ A.P. Ramirez, Annu. Rev. Mater. Sci. 24, 453 (1994).

${ }^{2}$ M. Shiga, H. Wada, Y. Nakamura, J. Deportes, and K.R.A. Ziebeck, J. Phys. Soc. Jpn. 57, 3141 (1988); M. Shiga, K. Fujisawa, and H. Wada, ibid. 62, 1329 (1993).

${ }^{3}$ B.D. Gaulin, J.N. Reimers, T.E. Mason, J.E. Greedan, and Z. Tun, Phys. Rev. Lett. 69, 3244 (1992).

${ }^{4}$ M.J. Harris, M.P. Zinkin, Z. Tun, B.M. Wanklyn, and I.P. Swainson, Phys. Rev. Lett. 73, 189 (1994).

${ }^{5}$ D. Yanagishima and Y. Maeno, J. Phys. Soc. Jpn. 70, 2880 (2001)

${ }^{6}$ H. Fukazawa and Y. Maeno, Phys. Rev. B 67, 054410 (2003).

${ }^{7}$ Y.J. Uemura, A. Keren, K. Kojima, L.P. Le, G.M. Luke, W.D. Wu, Y. Ajiro, T. Asano, Y. Kuriyama, M. Mekata, H. Kikuchi, and K. Kakurai, Phys. Rev. Lett. 73, 3306 (1994).

${ }^{8}$ R. Coldea, D.A. Tennant, A.M. Tsvelik, and Z. Tylczynski, Phys. Rev. Lett. 86, 1335 (2001).

${ }^{9}$ R. Moessner and J.T. Chalker, Phys. Rev. Lett. 80, 2929 (1998).

${ }^{10}$ J.N. Reimers, Phys. Rev. B 45, 7287 (1992).

${ }^{11}$ B. Canals and C. Lacroix, Phys. Rev. Lett. 80, 2933 (1998).

${ }^{12}$ A.B. Harris, A.J. Berlinsky, and C. Bruder, J. Appl. Phys. 69, 5200 (1991).

${ }^{13}$ M. Isoda and S. Mori, J. Phys. Soc. Jpn. 67, 4022 (1998).

${ }^{14}$ Y. Yamashita and K. Ueda, Phys. Rev. Lett. 85, 4960 (2000).

${ }^{15}$ A. Koga and N. Kawakami, Phys. Rev. B 63, 144432 (2001).

${ }^{16}$ H. Tsunetsugu, J. Phys. Soc. Jpn. 70, 640 (2001); Phys. Rev. B 65, 024415 (2001).

${ }^{17}$ S.E. Palmer and J.T. Chalker, Phys. Rev. B 64, 094412 (2001).

${ }^{18}$ E.H. Lieb and P. Schupp, Phys. Rev. Lett. 83, 5362 (1999).

${ }^{19}$ O.A. Starykh, R.R.P. Singh, and G.C. Levine, Phys. Rev. Lett. 88, 167203 (2002)

${ }^{20}$ E. Berg, E. Altman, and A. Auerbach, cond-mat/0206384 (unpublished).

${ }^{21}$ J.-B. Fouet, M. Mambrini, P. Sindzingre, and C. Lhuillier, Phys. Rev. B 67, 054411 (2003).
${ }^{22}$ J.T. Chalker, P.C.W. Holdsworth, and E.F. Shender, Phys. Rev. Lett. 68, 855 (1992).

${ }^{23}$ I. Ritchey, P. Chandra, and P. Coleman, Phys. Rev. B 47, 15342 (1993).

${ }^{24}$ P. Lecheminant, B. Bernu, C. Lhuillier, L. Pierre, and P. Sindzingre, Phys. Rev. B 56, 2521 (1997).

${ }^{25}$ O. Tchernyshyov, R. Moessner, and S.L. Sondhi, Phys. Rev. Lett. 88, 067203 (2002).

${ }^{26}$ S. Kondo, D.C. Johnston, C.A. Swenson, F. Borsa, A.V. Mahajan, L.L. Miller, T. Gu, A.I. Goldman, M.B. Maple, D.A. Gajewski, E.J. Freeman, N.R. Dilley, R.P. Dickey, J. Merrin, K. Kojima, G.M. Luke, Y.J. Uemura, O. Chmaissem, and J.D. Jorgensen, Phys. Rev. Lett. 78, 3729 (1997).

${ }^{27}$ S. Fujimoto, Phys. Rev. B 64, 085102 (2001).

${ }^{28}$ M. Isoda and S. Mori, J. Phys. Soc. Jpn. 69, 1509 (2000).

${ }^{29}$ S. Fujimoto, Phys. Rev. B 65, 155108 (2002).

${ }^{30}$ H. Tsunetsugu, J. Phys. Soc. Jpn. 71, 1844 (2002).

${ }^{31}$ Y. Yamashita and K. Ueda, Phys. Rev. B 67, 195107 (2003).

${ }^{32}$ J. Hopkinson and P. Coleman, Phys. Rev. Lett. 89, 267201 (2002).

${ }^{33}$ R. Shindou and N. Nagaosa, Phys. Rev. Lett. 87, 116801 (2001).

${ }^{34}$ Y. Taguchi, Y. Oohara, H. Yoshizawa, N. Nagaosa, and Y. Tokura, Science 291, 2573 (2001).

${ }^{35}$ T. Takeda, M. Nagata, H. Kobayashi, R. Kanno, Y. Kawamoto, M. Takano, T. Kamiyama, F. Izumi, and A.W. Sleight, J. Solid State Chem. 140, 182 (1998).

${ }^{36}$ D. Mandrus J.B. Thompson, R. Gaal, L. Forro, J.C. Bryan, B.C. Chakoumakos, L.M. Woods, B.C. Sales, R.S. Fishman, and V. Keppens, Phys. Rev. B 63, 195104 (2001).

${ }^{37}$ T. Furubayashi, T. Matsumoto, T. Hagino, and S. Nagata, J. Phys. Soc. Jpn. 63, 3333 (1994).

${ }^{38}$ M. Isobe and Y. Ueda, J. Phys. Soc. Jpn. 71, 1848 (2002).

${ }^{39}$ F. Ishii and T. Oguchi, J. Phys. Soc. Jpn. 69, 526 (2000). 
${ }^{40}$ A. Mielke, J. Phys. A 24, L73 (1991); 24, 3311 (1991).

${ }^{41}$ K. Kusakabe and H. Aoki, Phys. Rev. Lett. 72, 144 (1994).

${ }^{42}$ N. Biggs, Algebraic Graph Theory (Cambridge University Press, Cambridge, 1974).

${ }^{43}$ S. Fujimoto, Phys. Rev. Lett. 89, 226402 (2002).

${ }^{44}$ R. Shankar, Rev. Mod. Phys. 66, 129 (1994).

${ }^{45}$ C. Halboth and W. Metzner, Phys. Rev. B 61, 7364 (2000).

${ }^{46}$ P. Kopietz and T. Busche, Phys. Rev. B 64, 155101 (2001).

${ }^{47}$ D. Zanchi and H.J. Schulz, Phys. Rev. B 61, 13609 (2000).

${ }^{48}$ C. Honerkamp, M. Salmhofer, N. Furukawa, and T.M. Rice, Phys. Rev. B 63, 035109 (2001).

${ }^{49}$ J. Polchinski, Nucl. Phys. B 231, 269 (1984).

${ }^{50}$ C. Wetterich, Phys. Lett. B 301, 90 (1993).

${ }^{51}$ T.M. Morris, Int. J. Mod. Phys. A 14, 2411 (1994).
${ }^{52}$ M. Salmhofer, Renormalization (Springer, Berlin, 1998).

${ }^{53}$ Equation (4) in Ref. 43 should be corrected as Eq. (27) in this paper. This correction does not affect the main results in Ref. 43 qualitatively, but gives a quantative change in the estimation of $T_{c}$.

${ }^{54}$ B.I. Halperin and T.M. Rice, Solid State Phys. 21, 115 (1968).

${ }^{55}$ K. Matsuno, T. Katsufuji, S. Mori, Y. Moritomo, A. Machida, E. Nishibori, M. Takata, M. Sakata, N. Yamamoto, and H. Takagi, J. Phys. Soc. Jpn. 70, 1456 (2001).

${ }^{56} \mathrm{H}$. Harima (private communication).

${ }^{57}$ H. Sakai, M. Kato, K. Yoshimura, and K. Kosuge, J. Phys. Soc. Jpn. 71, 422 (2002).

${ }^{58}$ D.J. Singh, P. Blaha, K. Schwarz, and J.O. Sofo, Phys. Rev. B 65, 155109 (2002). 